\title{
Count-based method for specific binding ratio calculation in [I-123]FP- CIT SPECT analysis
}

\author{
Mahmudur G. M. Rahman ${ }^{1} \cdot$ Muhammad M. Islam $^{1,2} \cdot$ Tetsuya Tsujikawa $^{1} \cdot$ Yasushi Kiyono $^{1} \cdot$ Hidehiko Okazawa $^{1}$ (i)
}

Received: 7 August 2018 / Accepted: 2 September 2018 / Published online: 1 October 2018

(c) The Author(s) 2018

\begin{abstract}
Objective To calculate the specific binding ratio (SBR) appropriately in dopamine transporter (DAT) imaging, a method for extracting the striatal volume of interest (VOI) was developed.

Methods This study included 200 patients (72 \pm 10 years) who were suspected of parkinsonian syndromes (PS) or dementia with Lewy body (DLB). The patients were divided into three groups of PS with dopaminergic degeneration, DLB and nonPS after $\left[{ }^{123}\right.$ I] ioflupane (FP-CIT) SPECT and clinical follow-up. The image data were reconstructed with CT attenuation correction and scatter correction, and with only CT attenuation correction (CTAC). The new method extracted striatal VOI according to the high-level counts and the average striatum volume, and calculated SBR using the reference occipital counts. The SBR values for each patient were obtained using the Tossici-Bolt method $\left(\mathrm{SBR}_{\mathrm{Bolt}}\right)$ and our method. Reproducibility of SBR calculation using our method was compared by two operators.

Results The mean SBR values for the PS and DLB groups were significantly different from that of the non-PS group with both methods. The coefficients of variation of the SBR were significantly smaller with the proposed method compared with those of $\operatorname{SBR}_{\text {Bolt }}(p<0.001)$, except for the CTAC images. There were no differences in SBR between the two operators using our method. The diagnostic accuracies with our method for the PS and DLB groups were 98.4 and $96.0 \%$, respectively. Conclusion Our new method for SBR calculation in the FP-CIT SPECT showed less coefficients of variation with high reproducibility, which would be useful for clinical diagnosis and in assessing the severity of diseases in follow-up studies.
\end{abstract}

Keywords [I-123]ioflupane (FP-CIT) SPECT · DAT · SBR · ACSC · CTAC

\section{Introduction}

Dopamine transporter (DAT) imaging using $\left[{ }^{123} I\right]$ ioflupane (FP-CIT) SPECT is widely used for clinical assessment of the nigrostriatal function in patients with parkinsonian syndromes (PS) [1-4]. To evaluate presynaptic functional reduction of the striata, the specific binding ratio (SBR), a semi-quantitative value of FP-CIT accumulation, is calculated using various methods. Many quantitative methods have been proposed to assist with visual image interpretation in cases of movement disorder with parkinsonism because visual evaluation relies on the observer's ability and may

Hidehiko Okazawa

okazawa@u-fukui.ac.jp

1 Biomedical Imaging Research Center, University of Fukui, 23-3, Matsuoka-Shimaizuki, Eiheiji, Fukui 910-1193, Japan

2 Department of Biomedical Engineering, Khulna University of Engineering and Technology, Khulna, Bangladesh induce inter- and intra-observer inconsistency [5]. Semiquantitative evaluation would be useful for assessing disease progression, evaluating disease severity, and observing the clinical outcome after treatment [6-8]. Since a method with manual region of interest (ROI) selection revealed intra- and inter-observer variability [5], a three-dimensional (3D) automatic semi-quantification method was developed and used for data analysis in multicenter studies [9]. A 3D-ROI semiquantification software program named 'BasGan' is now very commonly used in Europe [9, 10]; the shape and location of the basal ganglia are automatically determined based on the Talairach atlas. Another sophisticated method, the Tossici-Bolt (TB) method, is also widely used for SBR calculation [11]. In this method, a large size ROI including the striatum is selected to avoid the partial volume effect (PVE), and the intra- and inter-operator variability were reported to be small (3-4\%). However, the TB method provides 2- to 3 -fold greater SBR values compared with other methods, and thus a large variability with substantial overlapping in 
the semi-quantitative values of the striatal tracer accumulation between patient groups and healthy subjects.

In this study, a new method for extraction of the striatal volume of interest (VOI) was developed to calculate the SBR appropriately. The proposed method has a semi-automatic procedure with an easy and operator-friendly process for accurate measurement of SBR. The results of SBR with our method were compared with those from the TB method, the most common method for semi-quantitative SBR evaluation in Japan, and also evaluated for inert-operator variance.

\section{Materials and methods}

\section{Subjects}

Two hundred patients (91 males and 109 females, $72 \pm 10$ years) with suspected of PS or dementia with Lewy body (DLB) were included in this study. They were referred to the Departments of Neurology or Psychiatry in our University Hospital and underwent $\left[{ }^{123}\right.$ I]FP-CIT SPECT for differential diagnosis of movement disorder or dementia. The patients were divided into three groups, PS with dopaminergic degeneration $(n=101)$, DLB $(n=11)$ and non-PS $(n=88)$, after the $\left[{ }^{123}\right]$ FP-CIT SPECT, perfusion SPECT and clinical follow-up for more than 12 months. The PS group consisted of Parkinson's disease, multiple system atrophy, progressive supranuclear palsy and corticobasal degeneration/syndrome This study was designed retrospectively to improve the accuracy of SBR evaluation using FP-CIT SPECT data. The protocol of this study was approved by the Ethics Committee of our University, Faculty of Medical Sciences (\#20170225).

\section{SPECT imaging}

Patients underwent SPECT scans after intravenous slow injection of about $170 \mathrm{MBq}\left[{ }^{123} \mathrm{I}\right] \mathrm{FP}-\mathrm{CIT}$ in the morning (167 MBq at noon). The scan was started about 3-4 h after the injection using a SPECT/CT scanner (Symbia T2, Siemens, Erlangen, Germany) with a dual-head gamma camera and low- and medium-energy general-purpose (LMEGP) collimators. The settings of SPECT/CT scans were the same as in the previous study [12]. In brief, the gamma camera was calibrated for a $159-\mathrm{keV}$ photo-peak and $\pm 10 \%$ energy window. Two sub-windows for triple-energy window scatter correction (SC) were set as a 7\% energy window on the both upper and lower sides of the photo-peak window. SPECT data acquisition was performed in 45 frames with four cycles of $210 \mathrm{~s} /$ cycle scan for a $180^{\circ}$ angular range in steps of $4^{\circ}$. The two camera heads were positioned as close as possible to the patient's head. A low-dose CT scan for attenuation correction was performed after SPECT data acquisition.
SPECT data were reconstructed by an iterative algorithm using 3D-ordered subset expectation maximization (OSEM) with eight iterations and ten subsets. Two image sets were reconstructed from the image data, i.e., with $\mathrm{CT}$ attenuation correction and SC (ACSC), and with only CT attenuation correction and no SC (CTAC). After $1.5 \times$ expansion, the pixel size of each slice was $2.20 \mathrm{~mm}$ in a $128 \times 128$ matrix, and a 6.0-mm Gaussian filter was applied.

\section{New calculation method for SBR}

SBR can be defined as the ratio of striatal count concentration due to specific binding in the striatum to the non-specific binding in the background. The proposed method is based on the calculation of the specific radioactivity concentration from the most-intense voxel in the striatal region. First, the range of slices that included the bilateral striata was determined for each patient by visual observation. A large trapezoid VOI $\left(\mathrm{VOI}_{\mathrm{t}}\right)$ with adequate thickness completely covering the bilateral striata was determined first by drawing manually on the centre slice level (Fig. 1a), and then applied to all slices covering the whole striatum. This $\mathrm{VOI}_{t}$ was set only to indicate the striatal position, but not to be used for SBR calculation. The standard volume of the striatum is reported as $11.2 \mathrm{~mL}[11,13$, 14], which includes 1052 voxels for each side in the SPECT image with a voxel size of $2.2 \mathrm{~mm} \times 2.2 \mathrm{~mm} \times 2.2 \mathrm{~mm}$. We extracted consecutive 1052 high-intensity voxels from the maximal count of each side in the $\mathrm{VOI}_{\mathrm{t}}$ and saved this as the striatal VOI mask $\left(\mathrm{VOI}_{\mathrm{st}}\right)$ to obtain the bilateral striatal counts separately (Fig. 2). In this step, the right-side peak voxel was determined first from the right half area in the $\mathrm{VOI}_{\mathfrak{t}}$, followed by extraction of the left half peak voxel. VOIs for background activity $\left(\mathrm{VOI}_{\mathrm{bg}}\right)$ were manually selected in the occipital region using a couple of slices (Fig. 1b) and the $\mathrm{VOI}_{\text {bg }}$ mean count was obtained for the non-specific background count. The SBR

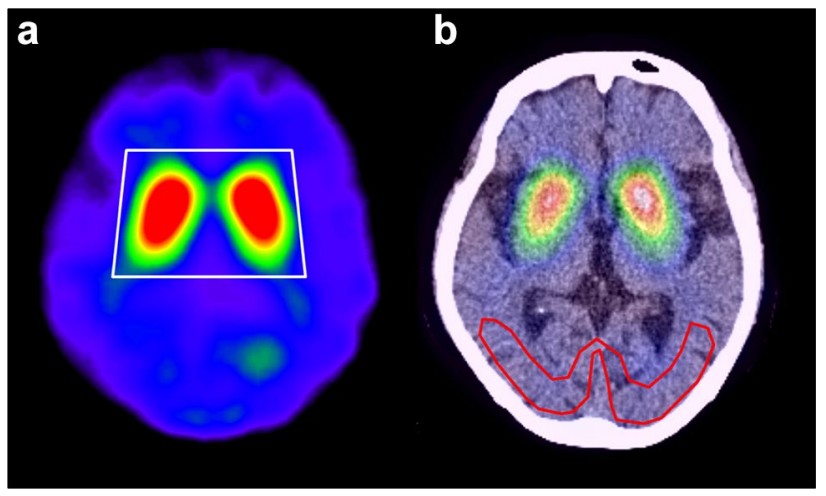

Fig. 1 Striatal trapezoid VOI (VOI $)$ location at the slice level of the striatal centre on DaT-SPECT (a). The VOI $_{t}$ includes the whole striatum for both sides. To obtain the background activity, the background VOI $\left(\mathrm{VOI}_{\mathrm{bg}}\right)$ was drawn on the occipital cortex using several image slices avoiding the sinus region and the cerebrospinal space (b) 
was calculated using the count concentration and the following equation:

$\mathrm{SBR}=\frac{\left[\left(\text { Total } \mathrm{VOI}_{\mathrm{st}} \text { count }\right) / 11.2-\left(\mathrm{VOI}_{\mathrm{bg}} \text { mean count }\right)\right](/ \mathrm{mL})}{\mathrm{VOI}_{\mathrm{bg}} \text { meancount }(/ \mathrm{mL})}$.

\section{Statistical analysis}

The SBR values obtained by the new method were compared with those from the TB method $\left(\mathrm{SBR}_{\text {Bolt }}\right)$ [11] obtained using a specific software program (DaT View, AZE Inc., Tokyo, Japan). Two operators separately analysed the DAT image data using our proposed method. In the PS group, the SBR values of the dominant side were used for analysis. In the DLB and non-PS groups, the average values of the bilateral SBR were compared. Statistical analysis was performed using SPSS Version 18.0, and $p<0.05$ was considered significant. Differences between groups were evaluated by the analysis of variance (ANOVA), and repeated measures ANOVA was applied for comparison of reconstruction methods. Both statistical analyses were followed by post hoc tests after detection of group differences. To evaluate difference in diagnostic accuracy using SBR values, receiver operating characteristic (ROC) analysis was used and area under the curve (AUC) was statistically compared using $\chi^{2}$-test.

\section{Results}

There were no differences in mean age ( $72 \pm 10$ years) among the three groups of PS, DLB and non-PS. Table 1 shows the $\mathrm{SBR}, \mathrm{SBR}_{\mathrm{Bolt}}$ values and coefficient of variation $(\mathrm{CV})$ for each group and the different methods for SBR calculation and image reconstruction. Patient groups showed significant differences in SBR when compared with the non-PS group $(p<0.0001)$ for

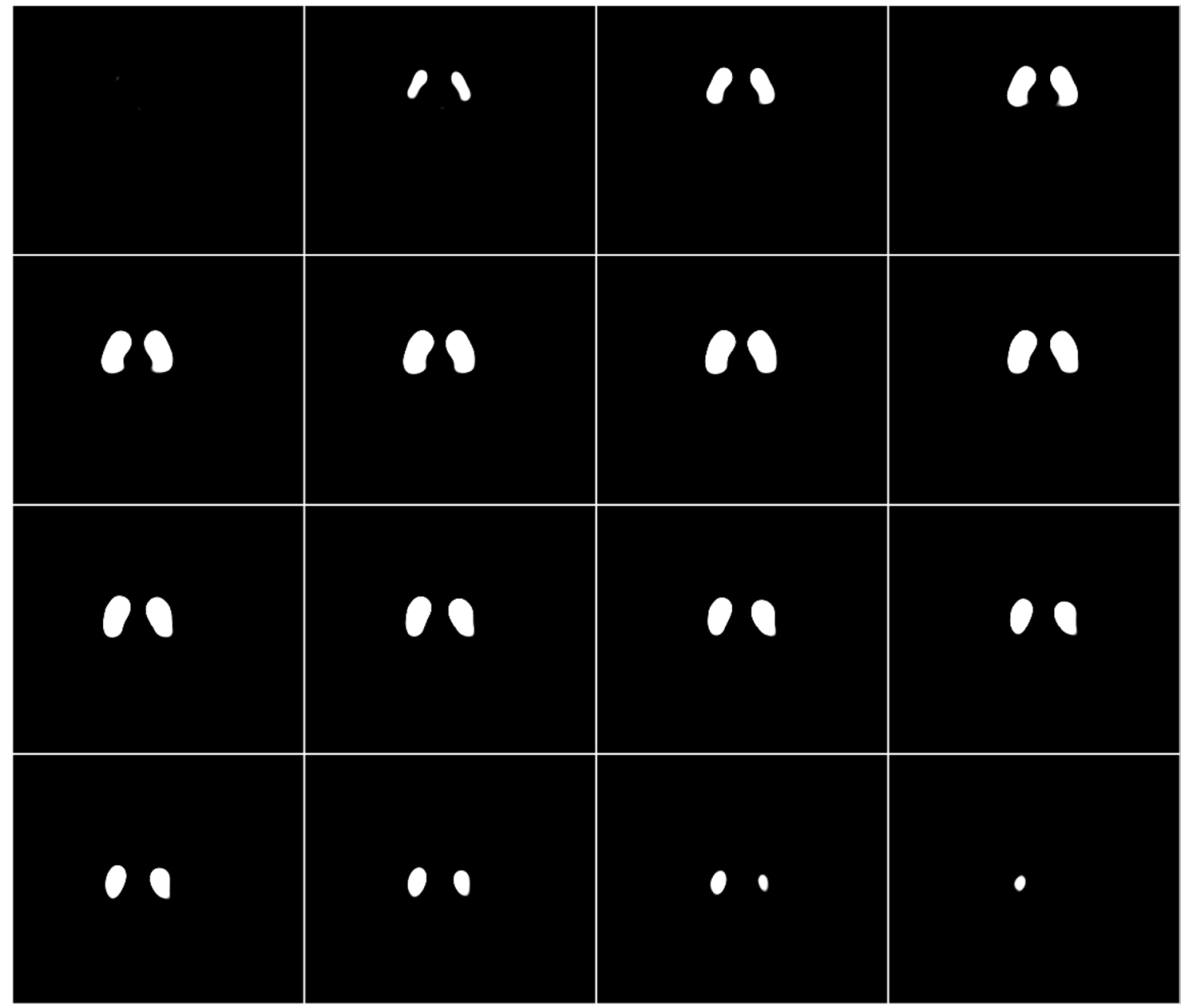

Fig. 2 Representative striatal VOI mask slices determined based on the intense voxel activity of the DAT image. A cluster of 1052 highintensity voxels $(11.2 \mathrm{~mL})$ was extracted consecutively from the maximal count of each side in the $\mathrm{VOI}_{\mathrm{t}}$ and saved as the striatal VOI mask $\left(\mathrm{VOI}_{\mathrm{st}}\right)$ to obtain the bilateral striatal counts 
Table 1 SBR values from two methods and two reconstruction images

\begin{tabular}{lllll}
\hline Groups & New-ACSC & New-CTAC & Bolt-ACSC & Bolt-CTAC \\
\hline PS $(n=101)$ & $1.05 \pm 0.45^{\dagger, *}$ & $0.97 \pm 0.37^{\dagger, *}$ & $2.78 \pm 1.41^{\dagger, *}$ & $2.49 \pm 1.17^{\dagger, *}$ \\
CV & 0.42 & 0.39 & 0.51 & 0.47 \\
DLB $(n=11)$ & $1.42 \pm 0.80^{\dagger, * *}$ & $1.20 \pm 0.65^{\dagger, * *}$ & $3.63 \pm 2.05^{\dagger, *}$ & $3.39 \pm 1.55^{\dagger, *}$ \\
CV & 0.56 & 0.54 & 0.57 & 0.46 \\
Non-PS $(n=88)$ & $2.97 \pm 0.54^{*}$ & $2.54 \pm 0.42^{*}$ & $7.48 \pm 1.72^{*}$ & $6.12 \pm 1.36^{*}$ \\
CV & 0.18 & 0.16 & 0.23 & 0.22 \\
\hline
\end{tabular}

Values are means $\pm \mathrm{SD}$

New- and Bolt- are SBR calculation methods; the proposed new method and the Tossici-Bolt method, ACSC and CTAC are reconstruction methods with or without scatter correction

$C V$ coefficient of variation

${ }^{\dagger} p<0.0001$ in comparison with non-PS group

${ }^{*} p<0.0001,{ }^{* *} p<0.01$ comparing ACSC and CTAC

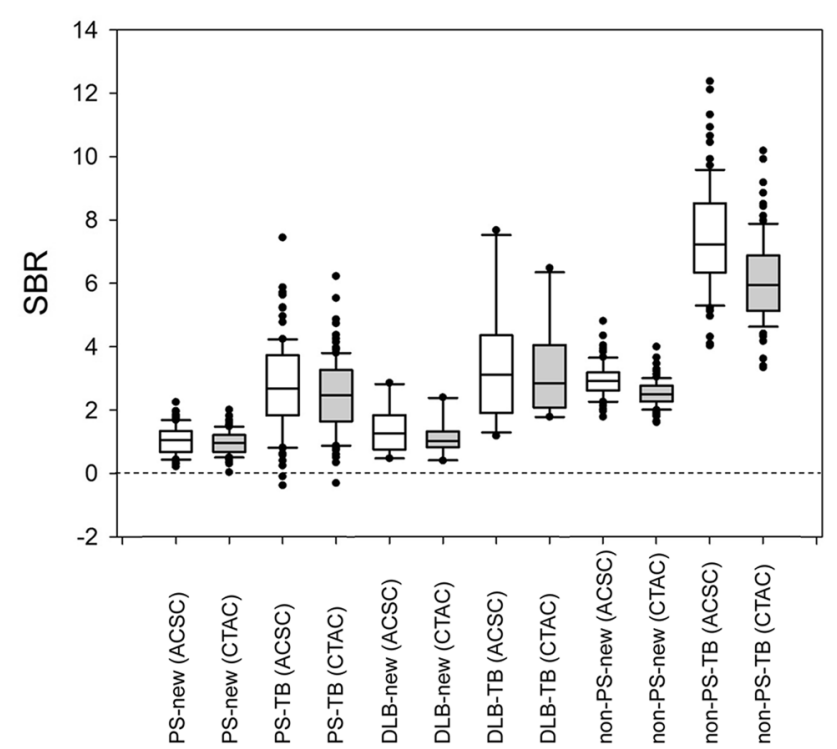

Fig. 3 Box-and-whisker plots for SBR values of the three groups (PS, DLB and non-PS) obtained by the two methods (our new method and TB method) and two reconstructions (ACSC and CTAC). PS and DLB groups showed significantly lower SBR than non-PS with both methods although the SBR values from our method were significantly lower than $\mathrm{SBR}_{\text {Bolt }}$ from the TB method

both our new method and the conventional TB method. The ACSC reconstruction showed significantly greater SBR values than the CTAC reconstruction with both methods. The TB method showed 2- to 3-fold greater SBR values ( $\mathrm{SBR}_{\mathrm{Bolt}}$ vs. SBR) as well as greater variance compared with the new method (Fig. 3; Table 1). The CVs of the SBR from the new method were significantly smaller compared with those of $\mathrm{SBR}_{\mathrm{Bolt}}$ $(p<0.001, F$ test), except for the CTAC reconstruction images in the DLB group. The repeated measures ANOVA between two operators showed no significant difference within the same reconstruction method according to our new method $(p>0.79)$. Figure 4 shows scatter plots comparing SBR values from the two

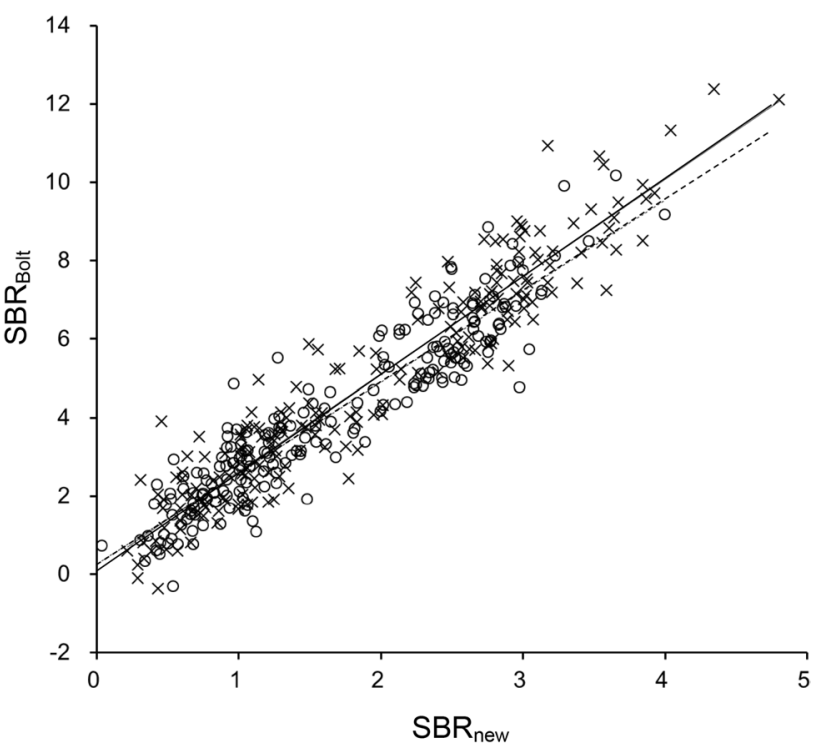

Fig. 4 Scatter plots of SBR values from the TB method $\left(\mathrm{SBR}_{\mathrm{Bolt}}\right)$ and our new method $\left(\mathrm{SBR}_{\text {new }}\right)$. Solid $(y=2.5 x+0.16, r=0.95)$ and dashed $(y=2.3 x+0.27, r=0.93)$ lines show good linear correlations between the two SBRs for ACSC (x) and CTAC (unfilled circles), respectively

methods. Both ACSC and CTAC images showed good linear correlations ( $r=0.95$ and 0.93 , respectively) between them.

ROC analysis for evaluation of diagnostic accuracy was performed and it showed greater AUC of the ROC curve in our new method $(>0.998)$ than that from the TB method $(<0.987)$ for both ACSC and CTAC images $(p<0.05$, Fig. 5). Taking cut-off values of SBR of 2.0 for ACSC and 1.8 for CTAC, which were determined by ROC analysis, the new method could distinguish the patient groups with a diagnostic accuracy of $98.4 \%$ for PS and $96.0 \%$ for DLB.

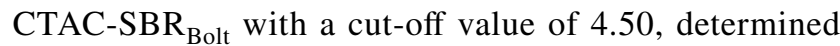
from a previous report [11], showed a diagnostic accuracy of $94.2 \%$ for our PS group and $91.8 \%$ for DLB. 


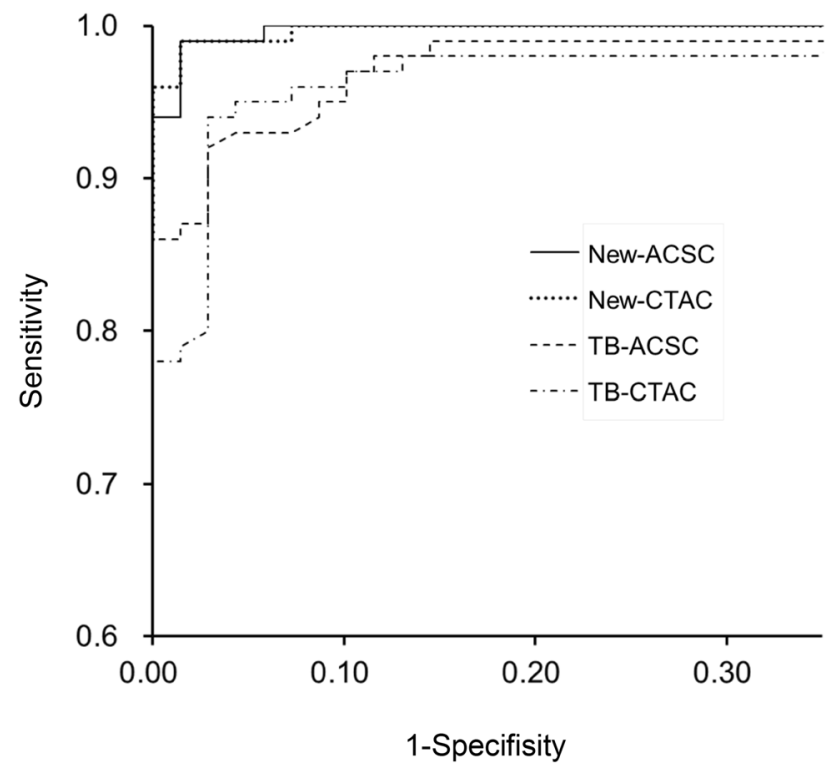

Fig. 5 ROC analysis for evaluation of diagnostic accuracy was performed and ROC curves showed greater AUCs of our new method (>0.998) compared with those of the TB method (ACSC 0.987, CTAC 0.983)

\section{Discussion}

DAT imaging using $\left[{ }^{123} \mathrm{I}\right] \mathrm{FP}-\mathrm{CIT}$ SPECT is very useful in the clinical setting to evaluate presynaptic striatal function in patients with parkinsonian syndrome and DLB $[3,15]$. Quantitative analysis has been expected as supporting information for clinical diagnosis, especially in borderline cases whose reduction of tracer accumulation is not definitive on visual observation. The SBR is widely used as a semiquantitative index for assessment of striatal dopaminergic deficit (SDD) [16, 17]. However, simplified methods such as the TB method sometimes provide unstable values although they may be able to avoid errors induced by the PVE [11, 18]. An automatic procedure may reduce observer variability caused by anatomical variations induced by atrophy or ventricular enlargement [5]. In this study, we proposed a new simplified method to easily obtain relatively stable SBR with good reproducibility. In the count-based method, the striatal VOI mask was well estimated by selecting the consecutive 1052 most-intense voxels based on the standard striatum size. An appropriate VOI mask selection method is a key to good reproducibility of SBR quantification. The new semiautomatic quantification method takes less time than the TB method and is significantly shorter than the manual method.

The SBR, the specific to non-specific radioactivity concentration, was calculated from the counts of extracted voxels $\left(\mathrm{VOI}_{\mathrm{st}}\right)$ and those of the reference region $\left(\mathrm{VOI}_{\mathrm{bg}}\right)$. In this study, the raw data were reconstructed into ACSC and CTAC image data, and compared among the three groups of PS, DLB and non-PS. The variability of SBR was smaller in the CTAC than the ACSC reconstruction in both methods because scatter correction includes greater variance in the regional counts. Although our method can be applied to non-AC reconstruction images, they were not included in the present study because of the greater variance in SBRs compared with AC images.

The large difference in SBRs between the TB method and our new method was caused simply by the difference in the calculation procedure. In the TB method, the $\mathrm{SBR}_{\mathrm{Bolt}}$ is calculated as follows:

$\operatorname{SBR}_{\text {Bolt }}=\left(Q_{\text {voi }} / C_{\mathrm{r}}-V_{\text {voi }}\right) / V_{\mathrm{s}}=\left(Q_{\mathrm{voi}}-C_{\mathrm{r}} V_{\mathrm{voi}}\right) / C_{\mathrm{r}} V_{\mathrm{s}}$, where $Q_{\text {voi }}$ is the total count of striatal VOI, $C_{\mathrm{r}}($ count $/ \mathrm{mL}$ ) is the count concentration of the reference region, and $V_{\text {voi }}$ and $V_{\mathrm{s}}$ are the volume of striatal VOI in the TB method and the average striatal volume $(11.2 \mathrm{~mL})[11,19]$. Since $Q_{\text {voi }}$ is equal to $C_{\text {voi }}$ (count concentration of striatal VOI) $\times V_{\text {voi }}$, the equation can be modified as follows:

$$
\begin{aligned}
\operatorname{SBR}_{\text {Bolt }} & =\left(C_{\text {voi }} V_{\text {voi }}\right) /\left(C_{\mathrm{r}} V_{\mathrm{s}}\right)-V_{\mathrm{voi}} / V_{\mathrm{s}} \\
& =\left(V_{\mathrm{voi}} / V_{\mathrm{s}}\right)\left[C_{\mathrm{voi}} / C_{\mathrm{r}}-1\right]=R \cdot\left[C_{\mathrm{voi}} / C_{\mathrm{r}}-1\right],
\end{aligned}
$$

where $R$ is a constant ratio of $V_{\text {voi }} / V_{\mathrm{s}}$. Because the size of $V_{\text {voi }}$ is fixed in the TB method (44 mm thickness), $\mathrm{SBR}_{\text {Bolt }}$ is usually greater than the SBR values from the manual method or other automatic method (Fig. 4). The TB method assumes that the striatal radioactivity of $\left[{ }^{123} \mathrm{I}\right] \mathrm{FP}-\mathrm{CIT}$ exists only inside the striata and the background outside them is almost homogeneous. The theory is perfectly correct under this assumption and $\mathrm{SBR}_{\mathrm{Bolt}}$ should be identical to other SBR values; however, in the real SPECT images, various factors affect the VOI counts. Since $\mathrm{C}_{\mathrm{voi}}$ is an average of heterogeneous regional counts affected by counts outside of the striatum including spillover, and PVE caused by inclusion of cerebrospinal fluid (CSF) space or other structures [20], $\left[C_{\mathrm{voi}} / C_{\mathrm{r}}-1\right]$ in the TB method is not necessarily identical to the SBR values from other methods. This is why the SBR values from the TB method were not exactly $R$-fold greater than those of our method; however, Fig. 4 clearly shows linear correlation between them. Furthermore, the large VOI in the TB method induces some fluctuation of $C_{\mathrm{voi}}$ depending on its location set by different operators, which is one of the reasons of unstable $\mathrm{SBR}_{\text {Bolt }}$ values compared with other semi-automatic method.

In our proposed method, the striatal mask determined in the $\mathrm{VOI}_{\mathrm{t}}$ consisted of the 1052 most-intense voxels for each side. Even in cases of reduced striatal accumulation like "dot pattern" and/or lateralized uptake, our method provides the same volume of striatal VOI at an appropriate location (Fig. 6). The mask may include outside structure of the striatum especially in reduced uptake cases; however, a cluster of the consecutive top 1052 voxels was included in the mask and there were no cases whose mask was located 
Fig. 6 An example of striatal VOI $\left(\mathrm{VOI}_{\mathrm{st}}\right)$ in a patient showing right dominant decrease with 'dot' pattern (a) shows somewhat round-shaped mask due to decrease in putaminal uptake (b). Each $\mathrm{VOI}_{\text {st }}$ consists of the same 1052 voxels

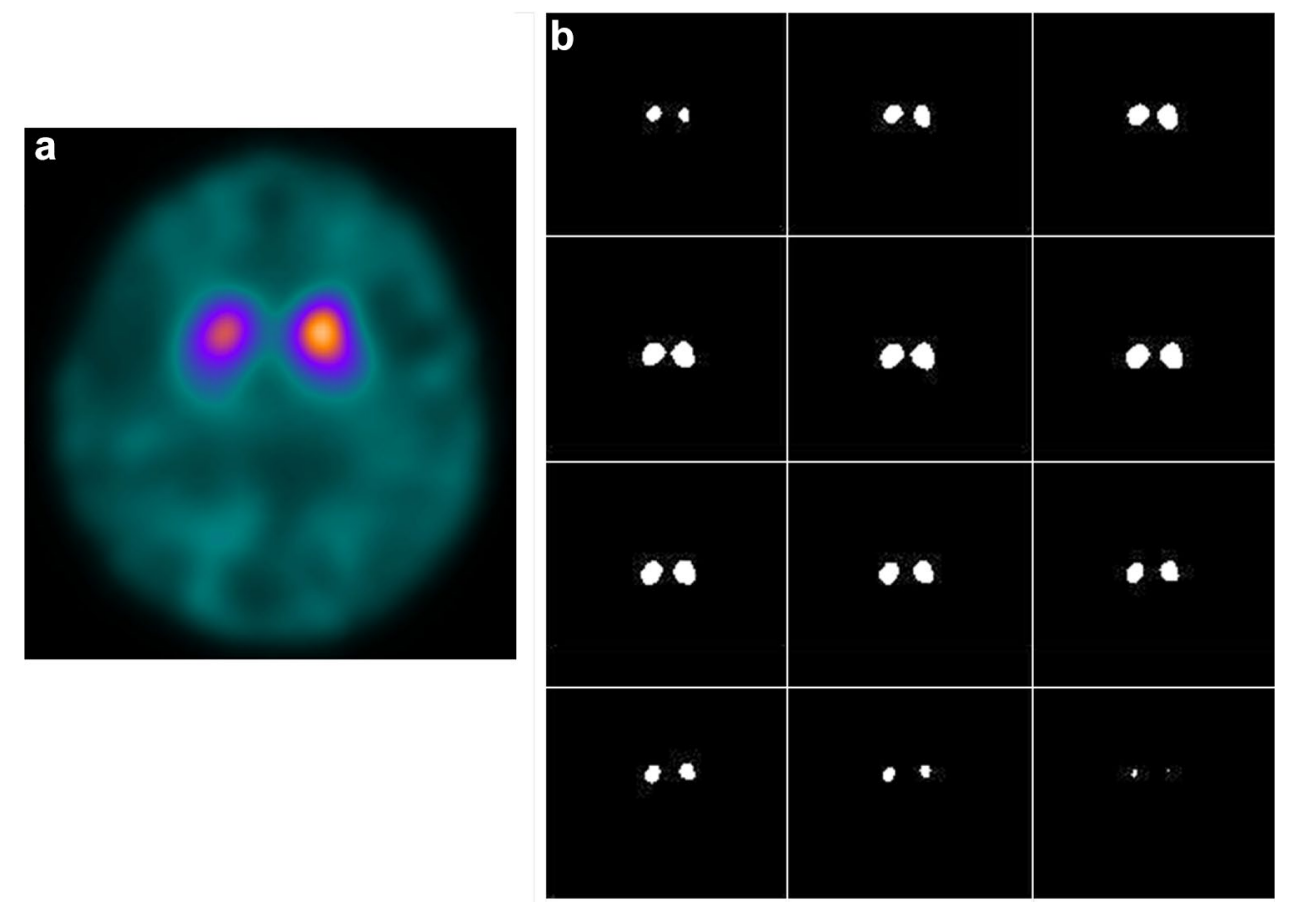

apart from the striatum despite very low striatal uptake. Furthermore, low accumulation similar to background does not affect SBR calculation as seen in the equation, because the low-intensity voxel values are almost identical to those of background. The method seems to be very practical and reasonable to place a VOI on the striatum. Recently, a new correction method for $\mathrm{SBR}_{\mathrm{Bolt}}$ calculation was introduced to avoid PVE from very low counts of the CSF space [21]. The method would reduce the influence of brain atrophy and infarction; however, it cannot avoid the effects of spillover as well as different size of individual $V_{\text {voi }}$ which induces variation of the constant $R$. Reduction of $V_{\text {voi }}$ provides smaller $R$ than the original one, inducing unstable enhancement factor in the TB method (see the equation above).

The variation in selection of reference regions may be another possible source of variability in SBR results. Researchers have defined various brain regions as the reference, such as the occipital lobes [22-27], the frontal lobes [28-30], and the cerebellum [31, 32]. The occipital lobe is relatively less sensitive to PVE and thereby PVE correction is not required [33]. In the proposed method, variability may be induced only by drawing reference region, although no significant difference was found between two different operators. Recent studies revealed stability in SBR calculation when selecting the whole cerebral VOI except for the striatum as the reference region [34]. In the present study, the difference was negligible between the different reference VOI (data not shown).

\section{Conclusion}

We proposed a new count-based method for SBR calculation in FP-CIT SPECT. This method can extract the striatal count in a simple way, with semi-automatic operation, and provided stable and reproducible results with less variation compared with previous simplified methods. The method will be very useful for calculating SBR in the clinical setting, especially in patient follow-up studies, as well as in multicentre studies.

Acknowledgements The authors thank Mr. Katsuya Sugimoto and the staff of the Biomedical Imaging Research Center and doctors in the Department of Neurology, Faculty of Medical Sciences, University of Fukui for technical and clinical support. This study was partly funded by a Grant-in-Aid for Scientific Research from the Japan Society for the Promotion of Science (18H02763).

\section{Compliance with ethical standards}

Conflict of interest The authors declare that they have no conflicts of interest.

Open Access This article is distributed under the terms of the Creative Commons Attribution 4.0 International License (http://creativeco mmons.org/licenses/by/4.0/), which permits unrestricted use, distribution, and reproduction in any medium, provided you give appropriate credit to the original author(s) and the source, provide a link to the Creative Commons license, and indicate if changes were made. 


\section{References}

1. Benamer HT, Patterson J, Wyper DJ, Hadley DM, Macphee GJ, Grosset DG. Correlation of Parkinson's disease severity and duration with ${ }^{123}$ I-FP-CIT SPECT striatal uptake. Mov Disord. 2000;15:692-8.

2. Tolosa E, Borght TV, Moreno E. Accuracy of DaTSCAN $\left({ }^{123}\right.$ I-ioflupane) SPECT in diagnosis of patients with clinically uncertain parkinsonism: 2-year follow-up of an open-label study. Mov Disord. 2007;22:2346-51.

3. Kägi G, Bhatia KP, Tolosa E. The role of DAT-SPECT in movement disorders. J Neurol Neurosurg Psychiatry. 2010;81:5-12.

4. Covington MF, Sherman S, Lewis D, Lei H, Krupinski E, Kuo $\mathrm{PH}$. Patient survey on satisfaction and impact of ${ }^{123}$ I-ioflupane dopamine transporter imaging. PLoS One. 2015;10(7):e0134457.

5. Koch W, Radau PE, Hamann C, Tatsch K. Clinical testing of an optimized software solution for an automated, observer independent evaluation of dopamine transporter SPECT studies. J Nucl Med. 2005;46:1109-18.

6. Chouker M, Tatsch K, Linke R, Pogarell O, Hahn K, Schwarz J. Striatal dopamine transporter binding in early to moderate advanced Parkinson's disease: monitoring of disease progression over 2 years. Nucl Med Commun. 2001;22:721-5.

7. Booij J, Speelman JD, Horstink MWIM, Wolters EC. The clinical benefit of imaging striatal dopamine transporters with [ $\left.{ }^{123} \mathrm{I}\right] \mathrm{FPCIT}$ SPET in differentiating patients with presynaptic parkinsonism from those with other forms of parkinsonism. Eur J Nucl Med. 2001;28:266-72.

8. Parkinson Study Group. Dopamine transporter brain imaging to assess the effects of pramipexole vs levodopa on Parkinson disease progression. JAMA. 2002;287:1653-61.

9. Calvini P, Rodriguez G, Inguglia F, Mignone A, Guerra UP, Nobili F. The basal ganglia matching tools package for striatal uptake semi-quantification: description and validation. Eur J Nucl Med Mol Imaging. 2007;34:1240-53.

10. Nobili F, Naseri M, De Carli F, Asenbaum S, Booij J, Darcourt $\mathrm{J}$, et al. Automatic semi-quantification of $\left[{ }^{123}\right.$ I]FP-CIT SPECT scans in healthy volunteers using BasGan version 2: results from the ENC-DAT database. Eur J Nucl Med Mol Imaging. 2013;40:565-73.

11. Tossici-Bolt L, Hoffmann S, Kemp P, Mehta R, Fleming J. Quantification of $\left[{ }^{123}\right.$ I]FP-CIT SPECT brain images: an accurate technique for measurement of the specific binding ratio. Eur $\mathrm{J}$ Nucl Med. 2006;33:1491-9.

12. Neishi H, Ikawa M, Okazawa H, Tsujikawa T, Arishima H, Kikuta K, Yoneda M. Precise evaluation of striatal oxidative stress corrected for severity of dopaminergic neuronal degeneration in patients with Parkinson's disease: a study with ${ }^{62} \mathrm{Cu}$-ATSM PET and ${ }^{123}$ I-FP-CIT SPECT. Eur Neurol. 2017;78:161-8.

13. Blinkov SM, Glezer II. The human brain in figures and tables. A quantitative handbook. New York: Basic Books; 1968. pp. $166-71$.

14. Aylward EH, Li Q, Habbak R, Warren A, Pulsifer MB, Barta PE, Jerram M, Pearlson G. Basal ganglia volume in adults with Down syndrome. Psychiatry Res. 1997;74:73-82.

15. Kupsch AR, Bajaj N, Weiland F, et al. Impact of DaTscan SPECT imaging on clinical management, diagnosis, confidence of diagnosis, quality of life, health resource use and safety in patients with clinically uncertain parkinsonian syndromes: a prospective 1-year follow-up of an open-label controlled study. J Neurol Neurosurg Psychiat. 2012;83:620-28.

16. Darcourt J, Booij J, Tatsch $\mathrm{K}$, et al. EANM procedure guidelines for brain neurotransmission SPECT using ${ }^{123}$ I-labelled dopamine transporter ligands, version 2. Eur J Nucl Med Mol Imaging. 2010;37:443-50.
17. Djang DS, Janssen MJ, Bohnen N, et al. SNM practice guideline for dopamine transporter imaging with ${ }^{123}$ I-ioflupane SPECT 1.0. J Nucl Med. 2012;53:154-63.

18. Tossici-Bolt L, Dickson JC, Sera T, de Nijs R, Bagnara MC, Jonsson C, et al. Calibration of gamma camera systems for a multicentre European ${ }^{123}$ I-FP-CIT SPECT normal database. Eur J Nucl Med Mol Imaging. 2011;38:1529-40.

19. Buchert R, Kluge A, Tossici-Bolt L, Dickson J, Bronzel M, Lange $C$, et al. Reduction in camera-specific variability in $\left[{ }^{123} \mathrm{I}\right]$ FP-CIT SPECT outcome measures by image reconstruction optimized for multisite settings: impact on age-dependence of the specific binding ratio in the ENC-DAT database of healthy controls. Eur J Nucl Med Mol Imaging. 2016;43:1323-36.

20. Nonokuma M, Kuwabara Y, Hida K, Tani T, Takano K, Yoshimitsu K. Optimal ROI setting on the anatomically normalized I-123 FP-CIT images using high-resolution SPECT. Ann Nucl Med. 2016;30:637-44.

21. Mizumura S, Nishikawa K, Murata A, Yoshimura K, Ishii N, Kokubo T, et al. Improvement in the measurement error of the specific binding ratio in dopamine transporter SPECT imaging due to exclusion of the cerebrospinal fluid fraction using the threshold of voxel RI count. Ann Nucl Med. 2018;32:288-96.

22. Walker Z, Costa DC, Walker RWH, Shaw K, Gacinovic S, Stevens $\mathrm{T}$, et al. CLE differentiation of dementia with lewy bodies from Alzheimer's disease using a dopaminergic presynaptic ligand. J Neurol Neurosurg Psychiatry. 2002;73:130-40.

23. Booij J, Tissingh G, Boer GJ, Speelman JD, Stoof JC, Janssen AGM, et al. $\left[{ }^{123}\right.$ I]FP-CIT SPECT shows a pronounced decline of striatal dopamine transporter labelling in early and advanced Parkinson's disease. J Neurol Neurosurg Psychiatry. 1997;62:133-40.

24. Booij J, Habraken JBA, Bergmans P, Tissingh G, Winogrodzka A, Wolters EC, et al. Imaging of dopamine transporter with iodine123-FP-CIT SPECT in healthy controls and patients with Parkinson's disease. J Nucl Med. 1998;39:1879-84.

25. Seibyl JP, Marek K, Sheff K, Zoghbi S, Baldwin RB, Charney DS, et al. Iodine-123- $\beta$-CIT and iodine-123-FPCIT SPECT measurement of dopamine transporter in healthy subjects and Parkinson's patients. J Nucl Med. 1998;39:1500-8.

26. van Dyck CH, Seibyl JP, Malison RT, Laurelle M, Wallace E, Zoghbi SS, et al. Age-related decline in striatal dopamine transporter binding with iodine-123- $\beta$-CIT SPECT. J Nucl Med. 1995;36:1175-81.

27. Badiavas K, Molyvda E, Iakovou I, Tsolaki M, Psarrakos K, Karatzas N. SPECT imaging evaluation in movement disorders: far beyond visual assessment. Eur J Nucl Med Mol Imaging. 2011;38:764-73.

28. Ichise M, Ballinger JR, Tanaka F, Moscovitch M, St. GeorgeHyslop PH, Raphael D, et al. Age-related changes in D2 receptor binding with iodine-123-iodobenzofuran SPECT. J Nucl Med. 1998;39:1511-8.

29. Schwarz J, Storch A, Koch W, Pogarelli O, Radau PE, Tatsch K. Loss of dopamine transporter binding follows a single exponential rather than linear decline. J Nucl Med. 2004;45:1694-7.

30. Tsuchida T, Ballinger JR, Vines D, Kim YJ, Utsunomiya K, Lang AE, et al. Reproducibility of dopamine transporter density measured with ${ }^{123}$ I-FPCIT SPECT in normal control and Parkinson's disease patients. Ann Nucl Med. 2004;18:609-16.

31. Pirker W, Asenbaum S, Bencsits G, Prayer D, Gerschlager W, Deecke L, et al. $\left[{ }^{123} \mathrm{I}\right]-\beta$-CIT SPECT in multiple system atrophy, progressive supranuclear palsy, and corticobasal degeneration. Mov Disord. 2000;15:1158-67.

32. Pirker W, Asenbaum S, Hauk M, Kandlhofer S, Tauscher J, Willeit $\mathrm{M}$, et al. Imaging serotonin and dopamine transporters with ${ }^{123}$ I-beta-CIT SPECT: binding kinetics and effects of normal aging. J Nucl Med. 2000;41:36-44. 
33. Crespo C, Gallego J, Cot A, Falcón C, Bullich S, Pareto D, Aguiar P, Sempau J, Lomeña F, Calviño F, Pavía J, Ros D. Quantification of dopaminergic neurotransmission SPECT studies with ${ }^{123}$ I-labelled radioligands. A comparison between different imaging systems and data acquisition protocols using Monte Carlo simulation. Eur J Nucl Med Mol Imaging. 2008;35:1334-42.
34. Kupitz D, Apostolova I, Lange C, Ulrich G, Amthauer H, Brenner W, et al. Global scaling for semi-quantitative analysis in FP-CIT SPECT. Nuklearmedizin. 2014;53:234-41. 